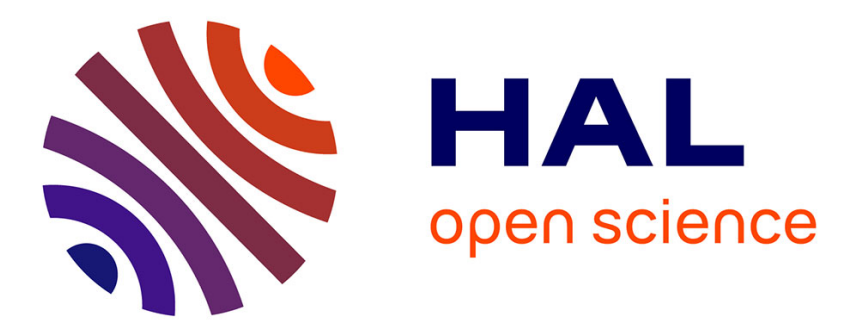

\title{
Traitement endovasculaire des anévrysmes de l'aorte thoracique descendante
}

\author{
Ludovic Canaud, Pierre Alric
}

\section{To cite this version:}

Ludovic Canaud, Pierre Alric. Traitement endovasculaire des anévrysmes de l'aorte thoracique descendante. La Presse Médicale, 2018, 47 (2), pp.153 - 160. 10.1016/j.lpm.2017.12.004 hal-01839258

\section{HAL Id: hal-01839258 \\ https://hal.umontpellier.fr/hal-01839258}

Submitted on 7 Jan 2020

HAL is a multi-disciplinary open access archive for the deposit and dissemination of scientific research documents, whether they are published or not. The documents may come from teaching and research institutions in France or abroad, or from public or private research centers.
L'archive ouverte pluridisciplinaire HAL, est destinée au dépôt et à la diffusion de documents scientifiques de niveau recherche, publiés ou non, émanant des établissements d'enseignement et de recherche français ou étrangers, des laboratoires publics ou privés. 


\section{Traitement endovasculaire des anévrysmes de l'aorte thoracique descendante}

Ludovic Canaud, Pierre Alric

CHU de Montpellier, hôpital Arnaud-de-Villeneuve, service de chirurgie thoracique et vasculaire, 191, avenue Doyen-Gaston-Giraud, 34090 Montpellier, France

Correspondance :

Ludovic Canaud, CHU de Montpellier, hôpital Arnaud-de-Villeneuve, service de chirurgie thoracique et vasculaire, 191, avenue Doyen-Gaston-Giraud, 34090 Montpellier, France.

ludoviccanaud@hotmail.com

\section{Points essentiels}

En vingt ans, le traitement endovasculaire des anévrysmes de l'aorte thoracique descendante est passé de la mise en place expérimentale d'une endoprothèse " home made », dans des anévrysmes anatomiquement favorables, à un traitement de première intention dans la plupart des cas. L'intérêt évident et immédiat de ces endoprothèses couvertes est d'éviter un geste chirurgical associé à une mortalité et à une morbidité élevée. Les améliorations technologiques continues des endoprothèses changent la place de cette technique dans le traitement des anévrysmes. Les études s'accordent à montrer une réduction du taux de morbidité et de mortalité périopératoire du traitement endovasculaire par rapport au traitement chirurgical conventionnel. D'un procédé réservé aux malades inopérables ou à haut risque chirurgical, il est maintenant scientifiquement démontré que cette approche constitue le traitement de première intention chez les patients présentant un anévrysme de l'aorte thoracique descendante, à condition qu'il présente une anatomie favorable. On observe toutefois l'apparition de complications spécifiques aux techniques endoluminales avec la description d'endofuites périprothétiques, de migrations, de fractures d'endoprothèse et de dissections rétrogrades. Le succès est associé à une sélection rigoureuse des patients grâce à un bilan préopératoire et au choix du type d'endoprothèse qui doit intervenir en préopératoire en fonction de la connaissance du matériel endovasculaire disponible. Le traitement endovasculaire des anévrysmes de l'aorte thoracique descendante impose d'avoir déterminé en préopératoire la stratégie de l'intervention.

\section{Key points}

Endovascular repair of the thoracic aorta

Degenerative aneurysms of the thoracic aorto are increasing in prevalence. The recognition of the decreased morbidity of this opproach compared with open repair was readily opparent, as it avoided left thoracotomy, aortic cross-clamping, and left heart bypass. Repair of isolated descending thoracic aortic aneurysms using stent grafts was introduced in 1995, and in an 
anatomically suitable subgroup of patients with thoracic aortic aneurysm, repair with endovascular stent graft provides fovorable outcomes, with decreased perioperative morbidity and mortality relative to open repair. The cornerstones of successful thoracic endovascular aneurysm repair are appropriate patient selection, thorough preprocedural planning, and cautious procedural execution. Since then, IEVAR is increasingly being used for other aortic pathologies such as complicated type $B$ dissection, traumatic aortic transection, and aneurysmal disease extending into the arch or visceral segment, requiring debranching procedures.

\section{Introduction}

Les anévrysmes de l'aorte thoracique ont une incidence de $6 / 100000$ par an.

Les anévrysmes de l'aorte thoracique représentent $20 \%$ des anévrysmes de l'aorte. La maladie athéromateuse représente l'étiologie la plus fréquente des anévrysmes de l'aorte thoracique. L'évolution vers la rupture étant inéluctables, le but du traitement est d'en prévenir ces conséquences fatales.

La technique chirurgicale pour le traitement des anévrysmes de l'aorte thoracique descendante s'est peu modifiée depuis le premier succès de De Bakey et Cooley en 1953 [1]. Elle nécessite une voie d'abord thoracique, une intubation sélective avec exclusion pulmonaire gauche, I'utilisation d'une assistance circulatoire et un clampage aortique proximal chez des patients souvent porteurs de comorbidités dans les pathologies dégénératives. Malgré l'amélioration des techniques chirurgicales et de la réanimation qui ont permis de diminuer la mortalité et la morbidité de cette approche, le remplacement chirurgical de l'aorte thoracique descendante est associé à un taux élevé de mortalité (5,7 \% à 15,1\% [2]) surtout pour les patients traités en urgence, les patients âgés et/ou à haut risque chirurgical. Le traitement endovasculaire, initialement mis en place pour le traitement des anévrysmes de l'aorte abdominale a été introduit comme une alternative moins invasive au traitement chirurgical conventionnel des anévrysmes de l'aorte descendante au début des années 90. Dake et al. [3] ont établi en 1994, la faisabilité du traitement endovasculaire des anévrysmes de l'aorte thoracique descendante par la mise en place d'endoprothèses couvertes auto-expansibles chez 13 patients; seuls $8 \%$ de l'ensemble de leurs patients porteurs d'un anévrysme de l'aorte thoracique étaient alors éligibles pour un traitement endovasculaire. En vingt ans, le traitement endovasculaire des pathologies de l'aorte thoracique est passé de la mise en place expérimentale d'une endoprothèse " home-made » dans des anévrysmes morphologiquement favorable, à un traitement établi faisant appel à des endoprothèses manufacturées et commercialisées pour le traitement de multiples pathologies aiguës ou chroniques de l'aorte thoracique, qu'il s'agisse d'anévrysmes, de faux anévrysmes, de dissections, de ruptures traumatiques, d'ulcérations, d'hématomes intramuraux ou d'aortites emboligènes. L'application de ces techniques endovasculaires a tout d'abord concerné les patients à haut risque chirurgical puis s'est élargie progressivement à l'ensemble des patients pour devenir désormais le traitement évoqué en première intention dans la plupart des cas. L'intérêt évident et immédiat de ces endoprothèses couvertes est d'éviter un geste chirurgical associé à une mortalité et une morbidité élevée. La plupart des études s'accordent à montrer une réduction des taux de morbidité et de mortalité périopératoire des traitements endovasculaires par rapport aux traitements chirurgicaux conventionnels $[4,5]$. On observe toutefois I'apparition de complications spécifiques aux techniques endoluminales avec la description d'endofuites périprothétiques, de migrations, de fractures d'endoprothèse et de dissections rétrogrades. La sélection rigoureuse des patients est une condition indispensable au succès du traitement endovasculaire des anévrysmes de l'aorte thoracique descendante. Cette étape cruciale impose la réalisation d'un bilan préopératoire avec une imagerie spécifique pour préciser l'accessibilité endovasculaire, la morphologie de la pathologie à traiter, et les mensurations de l'endoprothèse. Le choix d'une technique endovasculaire dans ces indications impose également de prévoir le déroulement du geste technique dans son intégralité. Par ailleurs, le choix du type d'endoprothèse doit intervenir en préopératoire en fonction de la connaissance du matériel endovasculaire disponible. Le traitement endovasculaire des anévrysmes de l'aorte thoracique descendante impose d'avoir déterminé en préopératoire la stratégie de l'intervention.

\section{Indication}

\section{Clinique}

Les patients porteurs d'un anévrysme de l'aorte thoracique doivent bénéficier d'une pris en charge chirurgicale lorsque l'anévrysme est compliqué, symptomatique et/ou volumineux (diamètre transversal maximal supérieur à $55 \mathrm{~mm}$ ) et/ou évolutif (croissance de au moins $5 \mathrm{~mm}$ en 6 mois) [6]. Certaines formes anatomiques ou étiologiques incitent également à intervenir sans tenir compte du diamètre de l'anévrysme : anévrysme sacciforme, post-traumatique, infectieux.

\section{Critères morphologiques}

Les critères morphologiques pour permettre une exclusion efficace d'un anévrysme de l'aorte thoracique descendante sont : 
- un collet proximal et distal sains de $15 \mathrm{~mm}$ de long. Une angulation modérée $<60^{\circ} \mathrm{C}$;

- la possibilité d'un accès vasculaire par voie fémorale, iliaque ou aortique pour l'introduction d'une endoprothèse avec un introducteur de 18 à $25 \mathrm{~F}$.

\section{Indication du traitement endovasculaire : rapport de la haute autorité de santé [7]}

Le rapport de la haute autorité de santé publié en 2006 conclut que le traitement endovasculaire de l'aorte thoracique semble apporter un bénéfice vraisemblable en termes de mortalité opératoire et de morbidité sévère, sous réserve d'une évaluation rigoureuse à moyen terme et si les conditions anatomiques ont été estimées favorables.

Ce traitement ne peut-être effectué que dans des centres ayant l'expertise des 2 traitements endovasculaires et chirurgicaux, et disposant d'un plateau technique adéquat. Les patients doivent être informés des avantages et des inconvénients des techniques de réparation disponibles que sont la chirurgie ouverte et le traitement endovasculaire.

\section{Autres indications du traitement endovasculaire de l'aorte thoracique}

Ruptures traumatiques de l'aorte thoracique descendante La survenue d'une rupture de l'aorte thoracique est le plus souvent liée à une décélération brutale lors d'un accident de haute vélocité. Du fait du mécanisme et de la haute vélocité de ces accidents, il s'agit le plus souvent de patients polytraumatisés associant des lésions cérébrales, rachidiennes, pulmonaires et digestives. Le traitement chirurgical conventionnel des ruptures de l'isthme est grevé d'une mortalité de 8 à $15 \%$ malgré l'utilisation d'une assistance circulatoire et les progrès de la réanimation [8].

Le taux moyen de mortalité globale de 4,9\% et surtout le faible taux moyen de mortalité liée à la rupture aortique de $1 \%$ de cette meta-analyse semblent prouver le bénéfice du traitement endovasculaire par rapport à la chirurgie conventionnelle associée à un taux de mortalité de 8 à $15 \%$ dans la revue de la littérature de Jahormi et al. [8] chez ces patients fragilisés par un polytraumatisme.

L'efficacité à court et moyen terme [9] du traitement endovasculaire des ruptures isthmiques traumatiques permet de proposer ce traitement en première intention chez ces patients polytraumatisés.

\section{Dissection de l'aorte thoracique descendante (type B selon la classification de Standford)}

Une dissection est une déchirure en général transversale de la couche intimale qui permet au flux sanguin de pénétrer dans le mur artériel. Un plan de clivage se crée entre l'intima et la média dans lequel le flux sanguin circule constituant un faux chenal. Les dissections sont dites aiguës lorsqu'elles sont identifiées dans les 15 jours qui suivent l'apparition de symptômes. Elles sont dites chroniques lorsque les symptômes datent de plus de 15 jours.

On distingue les dissections compliquées et les non-compliquées. Les dissections compliquées comprennent les ruptures, les augmentations de diamètre, l'extension de la dissection, une hypertension non contrôlable, des douleurs réfractaires, un syndrome de mal perfusion et/ou l'apparition d'anévrysme. Les dissections non compliquées sont celles indemnes des éléments précités.

Le but de l'endoprothèse aortique est de couvrir la (les) porte(s) d'entrée pour obtenir la thrombose du faux chenal. Ce faisant on espère réduire la pression dans le faux chenal avec l'espoir de stopper la progression de la dissection.

Dissections compliquées

À la phase aiguë : comme pour les lésions traumatiques de l'aorte thoracique, la diminution de la mortalité et de la morbidité par rapport au remplacement chirurgical sous assistance circulatoire de l'aorte thoracique, nous permet de considérer le traitement endovasculaire comme le traitement de première intention [10].

À la phase chronique : la dissection tend à cicatriser. La paroi aortique et le flap intimal se fibrosent. Le faux chenal peut rester perméable ou se thromboser. Cependant, dans $20 \%$ à $50 \%$ des cas l'aorte s'élargit progressivement et évolue vers un anévrysme. En ce qui concerne les dissections chroniques, un traitement est indiqué en cas d'évolution anévrysmale avec diamètre $>60 \mathrm{~mm}$ et/ou de caractère évolutif de l'anévrysme. Le traitement endovasculaire des dissections chroniques présente comme spécificité un taux bien plus élevé de réinterventions en comparaison au traitement des autres pathologies de l'aorte thoracique. La place du traitement endovasculaire pour les anévrysmes disséquants de l'aorte thoracique descendante est prometteuse, mais du fait du taux élevé de réinterventions, sa place reste débattue.

Dissections non compliquées

Deux essais prospectifs randomisés étudient actuellement les résultats du traitement endovasculaire des dissections non compliquées de type B associé au traitement médical optimal en comparaison au traitement médical optimal seul.

La première étude prospective randomisée (INSTEAD) [11] se focalisait sur le traitement des dissections chroniques non compliquées. Cette étude à démontrée une diminution de la mortalité aortique à 5 ans des patients traités préventivement par endoprothèse.

La deuxième étude prospective randomisée (ADSORB) [12] se focalisait sur le traitement des dissections aiguës non compliquées. Cette étude n'a pas démontré une diminution de la mortalité aortique à 1 ans des patients traités préventivement par endoprothèse. Les résultats à moyen terme n'ont pas été rapportés à l'heure actuelle.

Les données actuelles ne permettent pas de proposer un traitement préventif aux patients porteurs d'une dissection non 
compliquée. Il faudra attendre les résultats à moyen terme de ADSORB pour pouvoir proposer un traitement préventif à tous les patients.

\section{Bilan préopératoire objectif}

L'évaluation préopératoire est cruciale. Elle doit déterminer la faisabilité du traitement endovasculaire, la stratégie et le choix de l'endoprothèse ainsi que les mensurations de l'endoprothèse.

\section{Accès vasculaire}

Les problèmes d'accès vasculaire doivent être minutieusement explorés en préopératoire par un bilan morphologique complet de l'aorte et des axes iliaques.

\section{collet proximal et distal}

Le collet doit être $\geq 15 \mathrm{~mm}$ de long, et avoir un diamètre $\leq 40 \mathrm{~mm}$. De plus il doit être peu angulé et sans thrombus. Un collet $\geq 20 \mathrm{~mm}$ semble actuellement être un garant supplémentaire du succès de l'exclusion endovasculaire d'un anévrysme.

\section{Choix de l'endoprothèse : diamètre et longueur}

Le diamètre de l'endoprothèse doit être surdimensionné de 5 à $15 \%$ par rapport au diamètre du collet proximal. La longueur de l'endoprothèse doit être choisi en fonction de l'extension de l'anévrysme. Le choix du type d'endoprothèse doit dépendre des conditions anatomiques (angulation du collet proximal, distance par rapport au tronc céliaque... .) et des caractéristiques des endoprothèses thoraciques commercialisées.

\section{Méthode}

Les principales techniques d'imagerie actuellement disponibles sont l'angio-TDM, l'angio-TDM avec reconstructions 3D, l'artériographie et l'angio-IRM.

\section{Angio-IRM}

En dehors du cadre de l'urgence, I'angio-IRM au gadolinium permet à elle seule d'obtenir un bilan morphologique complet, en évitant une néphrotoxicité majeure chez les patients insuffisants rénaux [13]. L'hétérogénéité de qualité des angio-IRM et leur faible disponibilité en font pour l'instant un examen de seconde intention.

\section{Angio-TDM}

L'angio-TDM est actuellement le " gold-standard » pour la réalisation des mensurations préopératoires, préalablement à la mise en place d'une endoprothèse aortique [14]. La disponibilité et la rapidité de réalisation de cet examen constituent un atout évident. Un scanner 64 barrettes effectue une acquisition thoraco-abdomino-pelvienne en 15 à 20 secondes, avec une injection de $100 \mathrm{ml}$ de produit de contraste. Il est, cependant admis que la mesure des diamètres artériels peut être surestimée si la coupe étudiée n'est pas perpendiculaire à l'axe du vaisseau, et que la mesure des longueurs est également perturbée par les angulations et la tortuosité des vaisseaux.

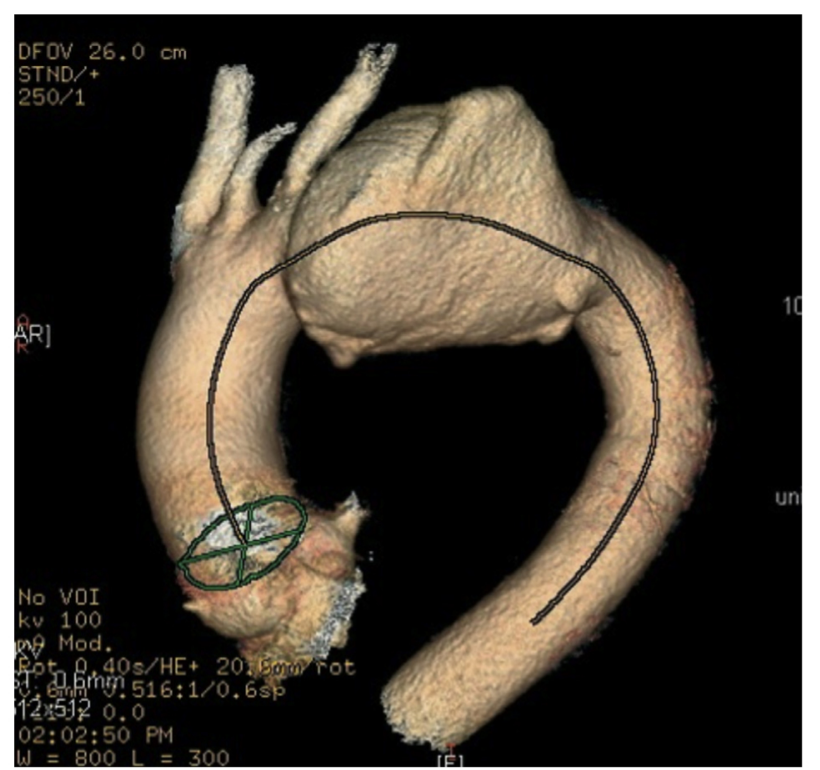

FIGURE 1

Angiotomodensitométrie (angio-TDM) avec reconstruction 30

L'analyse des images de l'angio-TDM et des reconstructions en 2D et 3D est effectuée grâce aux consoles de traitement (Figure 1).

\section{Artériographie}

L'artériographie donne d'excellentes informations sur les longueurs et la tortuosité vasculaire ; cependant, il n'est pas possible sur cet examen de mesurer de façon fiable les diamètres vasculaires ni de visualiser les calcifications aortiques ou un thrombus mural d'apposition.

\section{Endoprothèses}

Le choix du diamètre et de la longueur de l'endoprothèse aortique est directement corrélé aux mensurations aortiques réalisées sur le bilan morphologique préopératoire. La longueur de l'endoprothèse est fonction de l'extension en longueur sur l'aorte thoracique de la pathologie à traiter. Certaines endoprothèses sont proposées dans des longueurs de plus de $20 \mathrm{~cm}$. Il est possible de juxtaposer deux endoprothèses ou plus, avec un recouvrement d'environ $5 \mathrm{~cm}$ entre chacune d'entre elles.

\section{Techniques de pose}

\section{Plateau technique}

Nous réalisons le traitement endovasculaire de l'aorte thoracique au bloc opératoire pour différentes raisons.

Des conditions d'asepsie chirurgicale sont indispensables, car la contamination d'une endoprothèse peut avoir une issue dramatique.

\section{Anesthésie}

Une anesthésie générale est réalisée en routine pour permettre un déploiement optimal de l'endoprothèse en supprimant les 
mouvements parasites des malades souvent inconfortablement installé sur des tables opératoires rigides. II est toute fois possible de réaliser certaine de ces interventions à l'aide d'une anesthésie locale.

\section{Voies d'abord}

Abord chirurgical de l'artère fémorale :

Nous privilégions l'abord transversal $2 \mathrm{~cm}$ au-dessus de l'arcade crurale de l'artère fémorale pour diminuer le risque de lymphorrhée postopératoire. Un abord vertical est privilégié en cas de nécessité de revascularisation chirurgicale dans le même temps opératoire de l'artère fémorale.

Abord percutané :

Celle-ci est rendue possible grâce aux systèmes de fermeture comme le Prostar ${ }^{\circledR}$.

\section{Mise en place de l'endoprothèse}

L'aorte ascendante est cathétérisée sous contrôle scopique à l'aide d'un guide stiff.

\section{La méthode de déploiement est spécifique à chaque endoprothèse}

Un ballon compliant est utilisé pour parfaire l'apposition de la prothèse aux zones d'ancrage proximale et distale et au niveau du corps de l'endoprothèse.

Un contrôle angiographique est réalisé pour s'assurer de la bonne exclusion de la lésion, de détecter d'éventuelles endofuites précoces ou tardives et de s'assurer de la bonne perméabilité des troncs supra-aortiques ou du tronc cœliaque.

\section{Problèmes spécifiques \\ Problèmes d'accès}

Les problèmes sont liés à une tortuosité excessive des artères iliaques ou à des artères iliaques calcifiées, de petits calibres ou multi-sténosées.

Il existe des risques d'impossibilité de montée de l'endoprothèse, de rupture iliaque ou aortique.

\section{Collet proximal}

Les collets angulés et/ou courts $(<20 \mathrm{~mm})$ sont associés à un taux élevé d'échec de l'exclusion endovasculaire voire de complications engageant le pronostic vital.

Plusieurs techniques sont utilisées pour permettre un traitement des lésions nécessitant un ancrage proximal de l'endoprothèse dans la crosse aortique.

Traitement hybride de la crosse aortique

Pour obtenir un collet proximal satisfaisant, une approche hybride associant une transposition des troncs supra-aortiques suivi par l'exclusion endovasculaire de la lésion a été récemment proposée [15]. La transposition est réalisée pour créer une zone d'ancrage proximal (collet proximal) adéquate pour l'implantation l'endoprothèse tout en préservant la perfusion cérébrale et des membres supérieurs (Figures 2, 3 et 4).

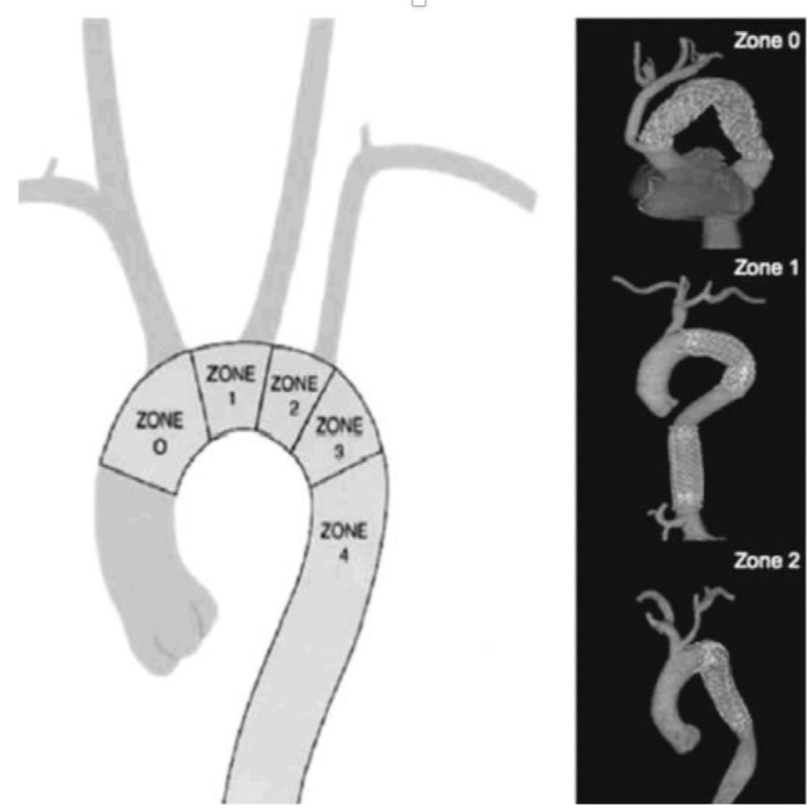

FIGURE 2

Classification des zones de la crosse aortique et angio TDM de contrôle après traitement endovasculaire de lésions aortiques en zone 0,1 et 2

Approche endovasculaire : endoprothèses branchées Une nouvelle génération d'endoprothèses aortiques a été développée afin de pouvoir réaliser un traitement exclusivement endovasculaire des lésions de la crosse aortique. Plusieurs modèles d'EDP destinés à exclure les anévrysmes de la crosse ont été proposés.

Déploiement de l'endoprothèse

Le risque de recul ou de basculement de l'endoprothèse lors du déploiement est le plus important au niveau de la crosse aortique. Il faut obtenir hypotension contrôlée induite.

\section{Ischémie Médullaire}

Le taux de paraplégie après mise en place d'une endoprothèse dans l'aorte thoracique descendante, toutes pathologies confondues, est compris entre $2 \%$ et 6,5\% [16]. En l'absence d'études randomisées contrôlées, il est difficile d'affirmer que ce taux est inférieur à celui observé après un traitement chirurgical conventionnel. Les raisons théoriques de ces taux relativement bas de paraplégie après traitement endovasculaire sont multiples : pas de clampage aortique, peu ou pas de périodes d'hypotension péri-opératoire en rapport avec une hypovolémie ou un saignement majeur.

\section{Accident vasculaire cérébral}

La manipulation des guides et de l'endoprothèse dans la crosse aortique fait que ces patients sont plus à risque d'accident vasculaire cérébral. 


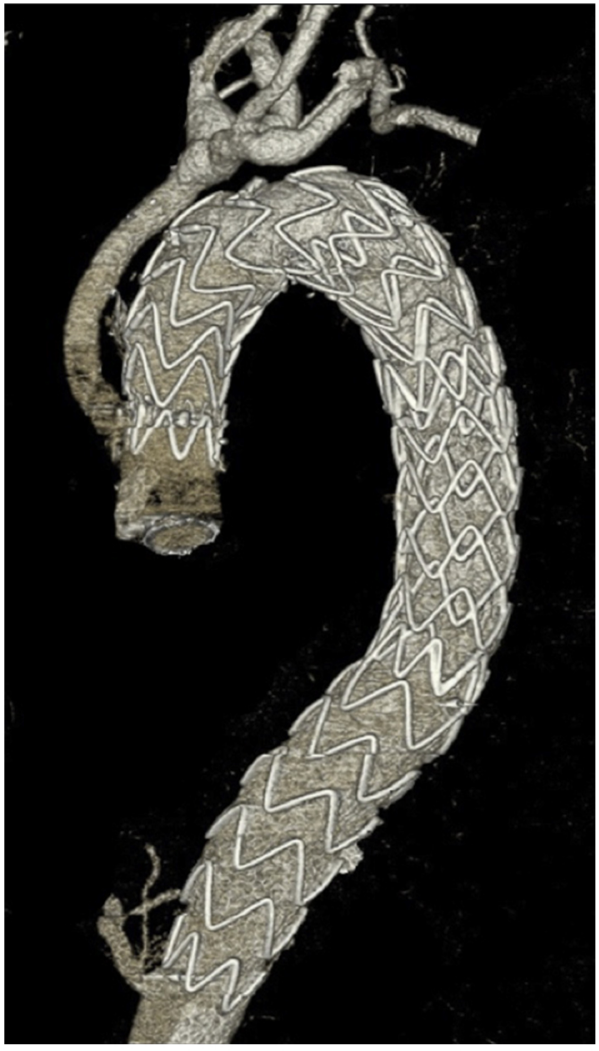

FIGURE 3

Angio TDM de contrôle après traitement endovasculaire d'un anévrysme en zone 0

Il faut au préalable étudier la perméabilité troncs supra-aortiques, rechercher la présence de plaques emboligènes au niveau de la crosse aortique. La présence de ce type de lésion doit faire discuter l'opportunité d'un traitement chirurgical conventionnel. La mise en place d'un traitement antiagrégant plaquettaire avant la chirurgie semble à recommander.

\section{Dissection rétrograde de l'aorte}

La survenue d'une dissection rétrograde de l'aorte thoracique est une complication redoutable possible du traitement endovasculaire de l'aorte thoracique. Son incidence moyenne (toutes pathologies confondues) est de 1,6\% [17]. Le délai entre l'implantation de l'endoprothèse et la survenue d'une dissection rétrograde est très variable, mais cette complication peut survenir 3 ans après la procédure endovasculaire. Le taux de mortalité associé à cette complication est très élevé (33,6\%). Une prise en charge chirurgicale urgente s'impose pour réaliser un remplacement de l'aorte ascendante et/ou de la crosse aortique sous circulation extracorporelle.

\section{Endofuites}

Une endofuite se définit par la persistance d'un flux sanguin circulant entre l'endoprothèse et l'anévrysme. Elle maintient

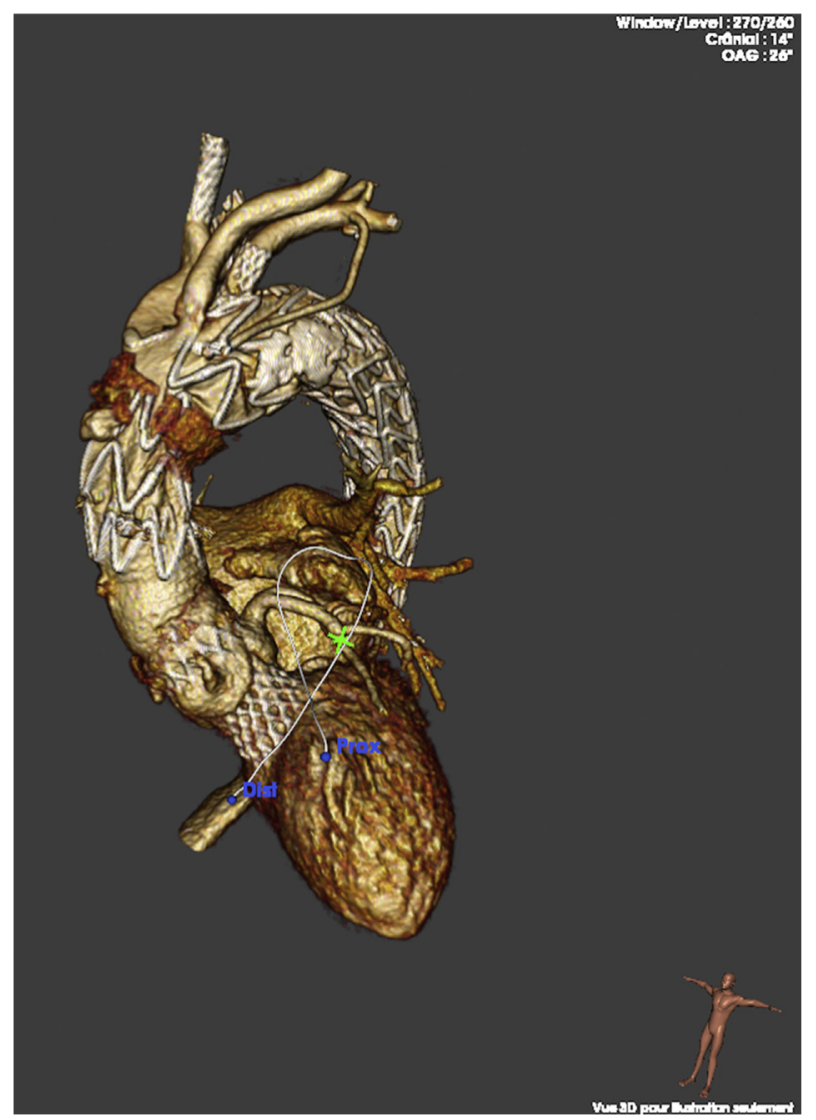

FIGURE 4

Angio TDM de contrôle après traitement endovasculaire d'un anévrysme en zone 0 avec une endoprothèse fenêtrée

une pression dans le sac anévrismal, source d'évolutivité, voire de rupture d'anévrysme.

Endofuite de type I

Elles sont soit proximales ou distales et sont les plus dangereuses : la pression dans le sac anévrismal reste égale à la pression systémique. Lorsqu'une endofuite de type I est associé à une expansion du sac anévrysmale, une prise en charge thérapeutique doit être systématique.

\section{Endofuite de type II}

Elles sont liées au reflux par les artères collatérales de la crosse aortique ou de l'aorte thoracique descendante : artère sous clavière gauche, artères intercostales, artères bronchiques oesophagiennes ou diaphragmatiques.

Les endofuites de type II sont moins fréquentes qu'à l'étage abdominal. Elles sont le plus souvent temporaires. La majorité des endofuites ne nécessitent aucun traitement mis à part une surveillance plus étroite.

\section{Endofuite de type III}

Elles sont liées à la déconnexion de deux endoprothèses et souvent lié à un défaut de recouvrement entre deux 
endoprothèses juxtaposée. La mise en place d'une nouvelle endoprothèse permet de régler ce type d'endofuite.

\section{Endofuite de type IV}

Les endofuites de type IV sont des fuites qui se produisent au niveau des sutures entre les composants métalliques qui constituent l'armature de la prothèse et la prothèse ou sont liées à des déchirures du polyester.

Endofuite de type $\mathbf{V}$

Les endofuites de types $V$ sont dues à une porosité excessive de la prothèse qui aboutit à la constitution d'un sérum périprothétique.

\section{Migration}

La migration distale de l'endoprothèse est moins fréquente à l'étage thoracique qu'a l'étage abdominale. Elle est le plus souvent liée au remodelage de l'aorte après traitement endovasculaire essentiellement à la dilatation du collet proximal. Les migrations sont favorisées par une apposition insuffisante de l'endoprothèse au niveau du collet proximal, en cas de sous dimensionnement du diamètre de la prothèse par rapport au diamètre du collet.

Les migrations ont pour conséquence l'apparition d'une des endofuites de type I.

Endoprothèses thoraciques et maladies du tissu conjonctif Les patients atteints du syndrome de Marfan ou d'autres maladies du tissu conjonctif méritent une attention particulière. II n'existe que quelques rapports de succès à court terme après traitement endovasculaire de l'aorte thoracique descendante chez les patients atteints du syndrome de Marfan [18-20]. Plusieurs complications ont été rapportées :

- dilatation de l'aorte malgré le mise en place de l'endoprothèse [18] ;

- rupture aortique lors de la mise en place de l'endoprothèse [21] ;

- dissection rétrograde [22].

Ces différentes complications font que le traitement endovasculaire chez les patients atteints du syndrome de Marfan ou de toute autre maladie du tissu conjonctif connue n'est pas recommandée, sauf si une intervention chirurgicale est clairement indiquée et que le risque la réparation chirurgicale conventionnelle est jugé prohibitif par un chirurgien.

\section{Complications infectieuses et fistules}

La survenue d'une infection, d'une fistule aorto-øsophagienne ou aorto-bronchique sont une complication rare, mais bien connue [23].

De nombreuses options thérapeutiques ont été proposées. La reconstruction vasculaire peut être in situ à l'aide d'une allogreffe ou par la réalisation d'un pontage extra-anatomique. La réparation oesophagienne ou pulmonaire en cas de fistule peut être conservatrice ou non.

Un traitement conservateur est associé de façon constante à une évolution fatale du fait d'une hémorragie massive ou d'une médiastinite [24].

\section{Surveillance}

Une surveillance prolongée clinique et radiologique est obligatoire et à vie. Les examens doivent être pratiqués à la sortie du patient ou dans le mois postopératoire ; puis au $3^{\mathrm{e}}$ mois en cas d'endofuite initiale ; puis au $6^{\mathrm{e}}$ et au $12^{\mathrm{e}}$ mois et annuellement. Le calendrier et les modalités de surveillance doivent être adaptés de l'existence ou non d'une anomalie et au risque de surexposition radiologique. Les modalités radiologiques de surveillance postopératoire sont selon les habitudes des équipes : scanner ou IRM.

La radiographie du thorax (clichés de face, de profil et de trois quarts) peut être utile pour dépister des anomalies des stents constituant l'armature de l'endoprothèse.

L'IRM présente comme avantage l'absence d'exposition du patient aux rayons $X$ et de pouvoir être réalisé chez les patients insuffisant rénaux.

\section{Résultats des études prospectives}

Les différentes compagnies ont mis en place des études prospectives pour évaluer les résultats de leurs endoprothèses. Toutes ces études sont non-randomisées [25-27]. En effet, le groupe chirurgical témoin incluait des patients dont plus de $50 \%$ avaient déjà été opérés avant le début de l'inclusion. Le taux de succès technique, le taux mortalité au $30^{\mathrm{e}}$ jour et à 1 an ainsi que le taux de " of paraplégie » étaient très larment inférieurs à ceux de la chirurgie conventionelle.

\section{Conclusion}

Le traitement endovasculaire des pathologies de l'aorte thoracique est dans des anévrysmes morphologiquement favorable, un traitement établi et évoqué en première intention dans la plupart des cas. L'intérêt évident et immédiat de ces endoprothèses couvertes est d'éviter un geste chirurgical associé à une mortalité et une morbidité élevée. La plupart des études s'accordent à montrer une réduction des taux de morbidité et de mortalité périopératoire des traitements endovasculaires par rapport aux traitements chirurgicaux conventionnels. On observe toutefois l'apparition de complications spécifiques aux techniques endoluminales avec la description d'endofuites péri-prothétiques, de migrations, de plicatures et de fractures d'endoprothèse. La sélection rigoureuse des patients est une condition indispensable au succès du traitement endovasculaire des pathologies de l'aorte thoracique descendante. Le traitement endovasculaire des pathologies de l'aorte thoracique descendante impose d'avoir déterminé en préopératoire la stratégie de l'intervention.

Déclaration de liens d'intérêts : Les auteurs déclarent ne pas avoir de liens d'intérêts. 


\section{Références}

[1] De Bakey ME, Cooley DA. Successful resection of aneurysm of thoracic aorta and replacement by graft. JAMA 1953;152:673-6.

[2] Stone DH, Brewster DC, Kwolek CJ, Lamuraglia GM, Conrad MF, Chung TK, et al. Stentgraft versus open-surgical repair of the thoracic aorta: mid-term results. J Vasc Surg 2006;44:1188-97.

[3] Dake MD, Miller DC, Semba CP, Mitchell RS, Walker PJ, Liddell RP. Transluminal placement of endovascular stent-grafts for the treatment of descending thoracic aortic aneurysms. N Engl I Med 1994;331:1729-34.

[4] Fairman RM, Criado F, Farber M, Kwolek C, Mehta M, White R, et al. Pivotal results of the Medtronic Vascular Talent Thoracic Stent Graft System: the VALOR trial. I Vasc Surg 2008:48:546-54.

[5] MatsumuraJS, Cambria RP, Dake MD, Moore RD, Svensson $L G$, Snyder S. TX2 clinical trial investigators. International controlled clinical trial of thoracic endovascular aneurysm repair with the Zenith TX2 endovascular graft: 1-year results. J Vasc Surg 2008;47:247-57.

[6] Svensson LG, Kouchoukos NT, Miller DC, Bavaria JE, Coselli JS, Curi MA, et al. Expert consensus document on the treatment of descending thoracic aortic disease using endovascular stent-grafts. Ann Thorac Surg 2008;85:1-41.

[7] Haute Autorité de Santé (HAS). Évaluation des endoprothèses dans le traitement des anévrysmes et des dissections de l'aorte thoracique 2016. www.has-sante.fr.

[8] Jahromi AS, Kazemi K, Safar HA, Doobay B, Cina C5. Traumatic rupture of the thoracic aorta: cohort study and systematic review. J Vasc Surg 2001;34:1029-34.

[9] Canaud L, Marty-Ané C, Ziza V, Branchereau P, Alric P. Minimum 10-year follow-up of endovascular repair for acute traumatic transection of the thoracic aorta. J Thorac Cardiovasc Surg 2015;149:825-9.

[10] Nienaber CA, Kische S, Ince H, Fattori R. Thoracic endovascular aneurysm repair for complicated type B aortic dissection. J Vasc Surg 2011;54:1529-33.

[11] Nienaber CA, Kische S, Rousseau H, Eggebrecht $\mathrm{H}$, Rehders $\mathrm{TC}$, Kundt $\mathrm{G}$, et al. Endovascular repair of type $B$ artic dissection: long-term results of the randomized investigation of stent grafts in aortic dissection trial. Circ Cardiovasc Interv 2013;6:407-11.

[12] Brunkwall J, Kasprzak P, Verhoeven E, Heijmen R, Taylor P. ADSORB Trialists. Endovascular repair of acute uncomplicated aortic type B dissection promotes aortic remodelling: 1 year results of the ADSORB trial. Eur J Vasc Endovasc Surg 2014;48:285-91.

[13] Neschis DG, Velazquez OC, Baum RA, et al. The role of magnetic resonance angiography for endoprosthetic design. I Vasc surg 2001;33:488-94.

[14] Parker MV, O'Donnell SD, Chang AS, et al. What imaging studies are necessary for abdominal aortic endograft sizing? A prospective blinded study using conventional computed tomography, aortography, and threedimensional computed tomography. J Vasc Surg 2005; 41:199-205.

[15] Canaud L, Hireche K, Berthet JP, Branchereau P, Marty-Ané C, Alric P. Endovascular repair of aortic arch lesions in high-risk patients or after previous aortic surgery: midterm results. J Thorac Cardiovasc Surg 2009.

[16] Bavaria JE, Appoo JJ, Makaroun MS, et al. Endovascular stent grafting versus open surgical repair of descending thoracic aortic aneurysms in low-risk patients: a multicenter comparative trial. J Thorac Cardiovasc Surg 2007; 133:285-8.

[17] Canaud L, Ozdemir BA, Patterson BO, Holt PJ, Loftus IM, Thompson MM. Retrograde aortic dissection after thoracic endovascular aortic repair. Ann Surg 2014;260:389-95.

[18] Nordon IM, Hinchliffe RJ, Holt PJ, Morgan R, Jahangiri M, Loftus IM, et al. Endovascular management of chronic aortic dissection in patients with Marfan syndrome. J Vasc Surg 2009;50:987-91
[19] Marcheix B, Rousseau H, Bongard V, Heijmen RH, Nienaber $C A$, Ehrlich $M$, et al. Stent grafting of dissected descending aorta in patients with Marfan's syndrome: mid-term results. JACC Cardiovasc Interv 2008;1:673-80.

[20] Geisbüsch P, Kotelis D, von Tengg-Kobligk H, Hyhlik-Dürr A, Allenberg JR, Böckler D. Thoracic aortic endografting in patients with connective tissue diseases. J Endovasc Ther 2008; 15:144-9.

[21] van Keulen JW, Moll FL, Jahrome AK, van Herwarden JA. Proximal aortic perforation after endovascular repair of a type B dissection in a patient with Marfan syndrome. J Vasc Surg 2009:50:190-2.

[22] Dong ZH, Fu WG, Wang YQ, Guo da Q, Xu X, Ji $Y$, et al. Retrograde type $A$ aortic dissection after endovascular stent graft placement for treatment of type B dissection. Circulation 2009;11:735-41.

[23] Czerny M, Zimpfer D, Fleck T, Gottardi R, Schoder M, Cejna M, et al. Successful treatment of an aortoesophageal fistula after emergency endovascular thoracic aortic stent-graft placement. Ann Thorac Surg 2005;80:1117-20.

[24] Eggebrecht H, Baumgart D, Radecke K, von Birgelen C, Treichel U, Herold U, et al. Aortoesophageal fistula secondary to stentgraft repair of the thoracic aorta. J Endovasc Ther 2004;11:161-7.

[25] Fairman RM, Tuchek JM, Lee WA, Kasirajan K, White $R$, Mehta $M$, et al. VALOR II investigators. Pivotal results for the Medtronic Valiant Thoracic Stent Graft System in the VALOR II trial. J Vasc Surg 2012;56:1222-31.

[26] Illig KA, Ohki T, Hughes GC, Kato M, Shimizu H, Patel HJ, et al. Zenith TX2 Low Profile study investigators. One-year outcomesfrom the international multicenter study of the Zenith Alpha Thoracic Endovascular Graft for thoracicendovascular repair. J Vasc Surg 2015;62:1485-94.

[27] Jordan WD, Rovin J, Moainie S, Bavaria J, Cambria R, Fillinger $M$, et al. Results of a prospective multicenter trial of CTAG thoracic endograft. J Vasc Surg 2015;61:589-95. 